\title{
LAGRANGIAN MECHANICS, GAUSS'S PRINCIPLE, QUADRATIC PROGRAMMING, AND GENERALIZED INVERSES: NEW EQUATIONS FOR NONHOLONOMICALLY CONSTRAINED DISCRETE MECHANICAL SYSTEMS
}

\author{
By \\ $\dagger$ ROBERT E. KALABA AND FIRDAUS E. UDWADIA \\ University of Southern California, Los Angeles, California
}

\begin{abstract}
In this paper we formulate Lagrangian mechanics as a constrained quadratic minimization problem. This quadratic minimization problem is then solved using the theory of generalized inverses of matrices thereby obtaining the explicit equations of motion of constrained, discrete mechanical systems. The approach extends the boundaries of Lagrangian mechanics in that we provide a general formulation for describing the constrained motion of such systems without either the use of Lagrange multipliers or the use of quasi-coordinates. An important feature of the approach is that we do not require prior knowledge of the specific set of constraints to accomplish this formulation. This makes the equations presented here more generally useful, and perhaps more aesthetic, than the Gibbs-Appell equations which require a felicitous choice of problem-specific quasi-coordinates. The new equations of motion presented here are applicable to both the holonomic and nonholonomic constraints that Lagrangian mechanics deals with. They are obtained in terms of the usual generalized coordinates used to describe the constrained system. Furthermore, they can be integrated by any of the currently available numerical integration methods, thus yielding analytical and/or computational descriptions of the motions of constrained mechanical systems.
\end{abstract}

Introduction. About a hundred years after Newton's Principia, Lagrange, in 1788 [1], presented his Mécanique Analytique, a book where he based the foundations of mechanics on the principles of virtual work and D'Alembert's principle. While Johann Bernoulli, Euler, and Leibnitz had made important contributions to this line of thinking, it was Lagrange who provided a general theory for constrained mechanical systems using the notion of generalized coordinates [1].

Lagrangian mechanics deals with constrained, discrete systems where the constraints are all equality constraints that can be represented in Pfaffian form [2, 3]. When these constraints are integrable, they are referred to as holonomic, otherwise

Received August 8, 1991.

1991 Mathematics Subject Classification. Primary 70F25, 70H35, 90C20.

${ }^{\dagger}$ Names of authors listed in alphabetical order.

(C)1994 Brown University 
as nonholonomic. Thus for a system of $N$ particles whose configuration is described by the $n$ coordinates $q_{1}, q_{2}, \ldots, q_{n}$, Lagrangian mechanics informs us that

$$
\sum_{s=1}^{n}\left\{\frac{d}{d t}\left(\frac{\partial T}{\partial \dot{q}_{s}}\right)-\frac{\partial T}{\partial q_{s}}-Q_{s}\right\} \delta q_{s}=0
$$

where the kinetic energy $T$ and the generalized forces $Q_{s}$ are, in general, functions of all the $q_{j}$ and $\dot{q}_{j}(j=1,2, \ldots, n)$ and of time $t$. The consistent constraints are described by the $m$ relations $(m<n)$

$$
\sum_{s=1}^{n} D_{r s} \dot{q}_{s}-D_{r}=0 \quad(r=1,2, \ldots, m),
$$

where $D_{r s}$ and $D_{r}$ are, in general, functions of $q_{j}(j=1,2, \ldots, n)$, and $t$. The equation set (2) may, in general, contain holonomic as well as nonholonomic constraints. When the $n$ coordinates that describe the system's configuration form a "generalized set of coordinates", the constraint equations (2) are all nonholonomic. This is because each of the holonomic constraints is used to eliminate one configuration coordinate thereby yielding the minimum number of coordinates needed to describe the system. Lagrange pointed out that the infinitesimal quantities, $\delta q_{s}$, in Eq. (1) are not arbitrary, but are constrained to be the virtual displacements of the mechanical system. These virtual displacements satisfy the following constraint equations:

$$
\sum_{s=1}^{n} D_{r s} \delta q_{s}=0 \quad(r=1,2, \ldots, m) .
$$

Thus, Eqs. (1), (2), and (3) form the basic equations of Lagrangian mechanics. We shall refer to this set as formulation L1.

The constraints on the infinitesimal displacements $\delta q_{s}$, represented by equation set (3), can be removed by the use of $m$ Lagrange multipliers, $\lambda_{r}(r=1,2, \ldots, m)$. Thus Eq. (3) can be incorporated into Eq. (1) yielding

$$
\sum_{s=1}^{n}\left\{\frac{d}{d t}\left(\frac{\partial T}{\partial \dot{q}_{s}}\right)-\frac{\partial T}{\partial q_{s}}-Q_{s}+\sum_{r=1}^{r=m} \lambda_{r} D_{r s}\right\} \overline{\delta q_{s}}=0,
$$

where now the quantities, $\overline{\delta q_{s}}$, are all entirely arbitrary. This then provides the following $n$ equations:

$$
\frac{d}{d t}\left(\frac{\partial T}{\partial \dot{q}_{s}}\right)-\frac{\partial T}{\partial q_{s}}-Q_{s}+\sum_{r=1}^{r=m} \lambda_{r} D_{r s}=0 \quad(s=1,2, \ldots, n)
$$

which, along with the constraint equations (2), describe the motion of the mechanical system. This alternative formulation which is also fundamental to Lagrangian mechanics, consisting of Eqs. (5) and (2), will be referred to as formulation L2.

We observe that while formulation L2 allows arbitrary displacements, $\overline{\delta q_{s}}$, formulation L1 does not. Yet, in formulation L2 the $m$ additional Lagrange multipliers, $\lambda_{r}$, need to be determined. We note therefore that formulation L2 relies on an expansion of the set of unknowns (i.e., the $q_{s}$ 's) to include the Lagrange multipliers. Thus 
formulation L2 provides a set of differential equations coupled to a set of nonintegrable constraint equations through the Lagrange multipliers. The elimination (or determination) of these Lagrange multipliers is by no means a simple affair, either analytically or computationally, when one is considering systems with many, many configuration coordinates and many constraints, i.e., systems with large $n$ and $m$. Also, there appears to be no general way of solving equation sets (5) and (2) simultaneously for arbitrary constraint equations. Determination of the Lagrange multipliers is dependent on the specific nature of the constraints thus leading to a case-by-case analysis of the constrained motion of nonholonomic systems. No general way of solving for them in terms of an as-yet-unspecified set of general constraint equations is possible.

An alternative approach for handling Lagrange formulation L1 was provided by Gibbs and Appell (working independently) in the late eighteen to early nineteenhundreds $[3,4]$. They expanded the set of coordinates used to describe the motion of nonholonomically constrained systems through the use of quasi-coordinates. These quasi-coordinates are not, in general, explicit functions of the original coordinates used to describe the motion of the nonholonomic system, but are expressed in nonintegrable, Pfaffian form. The set of Pfaffian equations defining the quasicoordinates and the set of constraint equations are then used jointly to eliminate all but the minimum number of coordinates needed to specify the dynamics of the system. The formulation results in the Gibbs-Appell equations $[3,4,5]$. While the quasi-coordinates may be freely chosen, their actual selection and the subsequent elimination procedure that follows are issues that depend critically on the specific nature of the problem at hand. Indeed, the Gibbs-Appell approach requires the following steps to obtain the equations of motion: (a) selecting the quasi-coordinates, (b) performing the subsequent eliminations and creating the Gibbs function, (c) differentiating the Gibbs function appropriately, and, (d) determining the generalized forces after elimination. No procedure to perform any of these steps, in a general setting, is available at present. This makes the Gibbs-Appell approach and the resultant formulation, once again, problem-specific, dependent on the specific nature of the constraint equations.

At the present time, this is the impasse at which Lagrangian mechanics is. As such, it precludes the rapid and systematic solution, both analytically and/or computationally, of problems that arise in many important areas of application such as multi-body dynamics, motion tracking-control of machine-tools, robotics, etc.

In this paper we show that by using the line of thinking pursued by Gauss when he derived the Principle of Least Constraint [6], the Lagrange formulation L1 can be recast to obtain a quadratic programming problem. This constrained quadratic minimization problem is solved explicitly using the theory of generalized inverses. Therefore, without the need for any Lagrange multipliers or the need for felicitously choosing quasi-coordinates that are problem specific, an explicit set of differential equations of motion is obtained. This set can then be analytically and/or computationally handled. In this paper, we thus provide an alternative approach to the analytical dynamics of discrete systems by developing the explicit equations (and a 
general formulation) for describing the motion of general, constrained dynamic systems. Like Lagrange's equations for holonomic systems, which can be formulated without prior knowledge of the specific generalized coordinates that are used, the equations obtained herein are formulated without the need for prior knowledge of the specific set of constraints.

We begin, in the next section, by recasting the $\mathrm{L} 1$ formulation into a quadratic minimization problem. The section that follows provides the solution to this minimization problem using generalized inverses. The next section attempts to link our new results with the L2 formulation, thereby exposing some deep connections and providing results of practical value for the control of mechanical systems so that they "follow" certain constraints. We then present two simple examples showing the efficacy of the approach, and end with a brief discussion of our new results and a comparison with earlier work.

Lagrangian mechanics as a quadratic minimization problem. We begin by recasting formulation $\mathrm{L} 1$ as a constrained minimization problem. We do this in the same vein as Gauss did in his original paper [6], albeit in a more general framework. The results we provide are new and not available in the usual treatises on analytical dynamics (e.g., in references $[3,5,7])$.

Consider the constrained system for which $q_{s}$ and $\dot{q}_{s}(s=1,2, \ldots, n)$ are known at time $t$. Given these quantities and the constraints (2) we can equivalently describe these constraints by differentiating Eq. (2) once to get the linearly independent relations

$$
\sum_{s=1}^{n} D_{r s} \ddot{q}_{s}-g_{r}=0 \quad(r=1,2, \ldots, m),
$$

where $g_{r}$ is, in general, a function of time $t$, and $q_{j}, \dot{q}_{j}(j=1,2, \ldots, n)$. Thus the trajectory $q_{s}(t)$ of our mechanical system satisfies Eqs. (1), (3), and (6).

Let us denote by a possible configuration of the system any configuration that satisfies the equations of constraint. Then, starting from the configuration already known at time $t$, denoted by $q_{s}$ and $\dot{q}_{s}$, we can describe the displacements, velocities, and accelerations related to this possible configuration by $q_{s}, \dot{q}_{s}$, and $\hat{\ddot{q}}_{s}$ $(s=1,2, \ldots, n)$. Since this is a possible configuration, it must satisfy the constraint equations

$$
\sum_{s=1}^{n} D_{r s} \hat{\ddot{q}}_{s}-g_{r}=0 \quad(r=1,2, \ldots, m) .
$$

Subtracting Eqs. (6) and (7) we obtain

$$
\sum_{s=1}^{n} D_{r s}\left(\hat{\ddot{q}}_{s}-\ddot{q}_{s}\right)=0 \quad(r=1,2, \ldots, m) .
$$

Since the quantities $\left(\hat{\dot{q}}_{s}-\ddot{q}_{s}\right)(s=1,2, \ldots, n)$ satisfy Eq. (3), they represent, by definition, a set of virtual displacements. Thus these possible acceleration changes from the true acceleration of the system (starting from the same time and system 
state) satisfy the same constraints as virtual displacements and need not be small. Thus we can recast the $\mathrm{L} 1$ formulation (specifically Eq. (1)) as

$$
\sum_{s=1}^{n}\left\{\frac{d}{d t}\left(\frac{\partial T}{\partial \dot{q}_{s}}\right)-\frac{\partial T}{\partial q_{s}}-Q_{s}\right\}\left(\hat{\tilde{q}}_{s}-\ddot{q}_{s}\right):=\sum_{s=1}^{n} Q_{s}^{\prime}\left(\hat{\ddot{q}}_{s}-\ddot{q}_{s}\right)=0,
$$

where $\hat{\dot{q}}_{s}(s=1,2, \ldots, n)$ are the accelerations that are relevant to any possible configuration of the system starting with the given $q_{s}$ and $\dot{q}_{s}$ at time $t$. Noting that the kinetic energy is a quadratic function in $\dot{q}_{s}$, it is always expressible as

$$
T=\frac{1}{2} \sum_{u=1}^{n} \sum_{v=1}^{n} a_{u v} \dot{q}_{u} \dot{q}_{v}+\sum_{u=1}^{n} b_{u} \dot{q}_{u}+c,
$$

where $a_{u v}, b_{u}$, and $c$ are, in general, functions of: (a) the time $t$, (b) the values of the point masses in the system, and (c) the configuration coordinates $q_{s}$, $s=1,2, \ldots, n$. Furthermore, the $n \times n$ matrix $A=\left[a_{u v}\right]$ is always symmetric and positive definite [2]. Denoting the vector $\left(q_{1} q_{2} \cdots q_{n}\right)^{\mathrm{T}}:=q$, the vector $\left(Q_{1} Q_{2} \cdots Q_{n}\right)^{\mathrm{T}}:=Q$, and the vector $\left(Q_{1}^{\prime} Q_{2}^{\prime} \cdots Q_{n}^{\prime}\right)^{\mathrm{T}}:=Q^{\prime}$, we can rewrite Eq. (9), using Eq. (10) (see, e.g., [2, pp. 221-223]) as

$$
(A \ddot{q}+f(q, \dot{q}, t)-Q)^{\mathrm{T}}(\hat{\ddot{q}}-\ddot{q}):=Q^{\prime \mathrm{T}}(\hat{\ddot{q}}-\ddot{q})=0,
$$

where $f$ and $Q$ are $n$-vectors that are each functions of $q, \dot{q}$, and $t$. Physically, the vector $Q^{\prime}$ is the generalized force exerted on the dynamic system due to the constraints (2), and relation (11) can be viewed as nothing but a restatement of D'Alembert's principle of virtual work.

Since relation (11) must be true for all possible configurations we next show that this leads to a minimum principle. We first present the following lemmas.

LeMMA 1. For any symmetric matrix $Y$, and any set of vectors $b, c$, and $\gamma$, we have

$$
(b-\gamma)^{\mathrm{T}} Y(b-\gamma)-(b-c)^{\mathrm{T}} Y(b-c)=(\gamma-c)^{\mathrm{T}} Y(\gamma-c)-2(b-c)^{\mathrm{T}} Y(\gamma-c) .
$$

Proof. This identity can be directly verified.

When $Y$ is positive definite, result (12) can be thought of geometrically as a generalization of the "cosine rule" in a triangle and can be expressed in terms of inner products as

$$
(b-\gamma, b-\gamma)_{Y}-(b-c, b-c)_{Y}=(\gamma-c, \gamma-c)_{Y}-2(b-c, \gamma-c)_{Y},
$$

where by $(u, v)_{Y}$ we mean $u^{\mathrm{T}} Y v$.

LEMMA 2. Any displacement vector, $(\hat{\ddot{q}}-\ddot{q})$, satisfies the relation

$$
\begin{aligned}
(A \hat{\ddot{q}}+ & f(q, \dot{q}, t)-Q)^{\mathrm{T}} A^{-1}(A \hat{\ddot{q}}+f(q, \dot{q}, t)-Q) \\
& -(A \ddot{q}+f(q, \dot{q}, t)-Q)^{\mathrm{T}} A^{-1}(A \ddot{q}+f(q, \dot{q}, t)-Q) \\
= & (\hat{\ddot{q}}-\ddot{q})^{\mathrm{T}} A(\hat{\ddot{q}}-\ddot{q})+2(A \ddot{q}+f(q, \dot{q}, t)-Q)^{\mathrm{T}}(\hat{\ddot{q}}-\ddot{q}),
\end{aligned}
$$

where $A$ is any symmetric positive-definite matrix. 
Proof. Noting that $A$ is a symmetric positive-definite matrix, in Eq. (12) set

$$
Y=A, \quad b=A^{-1}(Q-f), \quad c=\ddot{q}, \quad \text { and } \quad \gamma=\hat{q} .
$$

The result follows.

We next present the following result.

Result 1. For the virtual displacement vector $(\hat{\ddot{q}}-\ddot{q})$ that satisfies Eq. (11), the minimum value of

$$
G:=(A \hat{\ddot{q}}+f(q, \dot{q}, t)-Q)^{\mathrm{T}} A^{-1}(A \hat{\ddot{q}}+f(q, \dot{q}, t)-Q)
$$

given $q$ and $\dot{q}$ at time $t$, over all possible configurations, is obtained for $\hat{\ddot{q}}(t)=\ddot{q}(t)$. Noting the definition of $Q^{\prime}$, thus the dynamic system behaves as though it minimizes the measure of constraint given by

$$
G=\left(Q^{\prime}, Q^{\prime}\right)_{A^{-1}}
$$

at each instant of time. We will refer to $G$ as the Gaussian.

Proof. Using Lemma 2 and noting Eq. (11) we get

$$
\begin{aligned}
& (A \hat{\ddot{q}}+f(q, \dot{q}, t)-Q)^{\mathrm{T}} A^{-1}(A \hat{\ddot{q}}+f(q, \dot{q}, t)-Q) \\
& \quad-(A \ddot{q}+f(q, \dot{q}, t)-Q)^{\mathrm{T}} A^{-1}(A \ddot{q}+f(q, \dot{q}, t)-Q)=(\hat{\ddot{q}}-\ddot{q})^{\mathrm{T}} A(\hat{\ddot{q}}-\ddot{q}) .
\end{aligned}
$$

As the matrix $A$ is positive definite, the right-hand side of Eq. (18) is always positive and therefore the minimum of (16) occurs when $\hat{\dot{q}}(t)=\ddot{q}(t)$. Hence the actual motion of the constrained mechanical system is such as to minimize the constraint force, or Gaussian, $G$, as shown in Eq. (17).

We note that this minimum is a global minimum. The reasoning provided in this section while in the spirit of Gauss's original paper deviates substantially from it in that we do not need to consider the possible configurations over the infinitesimal time interval $(t, t+d t)$ as did Gauss. Furthermore, our results are applicable to systems whose configuration is described by generalized coordinates. Result 1 states the following: Given that we know the state (i.e., $q$ and $\dot{q}$ ) of a dynamic system at time $t$, of all the possible accelerations at that time consistent with the constraints, the actual one that the constrained dynamic system "chooses" is the one that minimizes the Gaussian, $G$, at that time.

This leads us to the conclusion that given the state of a constrained mechanical system at time $t_{0}$, the acceleration of the system at each instant of time beyond and including time $t_{0}$, must be such that it:

(1) minimizes $G$ given by Eq. (16) (or (17)) at each instant of time, and

(2) satisfies the set of constraint equations at each instant of time.

Thus we are led to a quadratic programming problem stated as follows: Given $q\left(t_{0}\right)$ and $\dot{q}\left(t_{0}\right)$, find $\ddot{q}\left(t_{0}\right)$ such that the Gaussian

(1) $G:=(A \ddot{q}+f(q, \dot{q}, t)-Q)^{\mathrm{T}} A^{-1}(A \ddot{q}+f(q, \dot{q}, t)-Q)$ is a minimum 
(2) $D \ddot{q}-g=0$,

where the $m \times n$ matrix $D=\left[D_{r s}\right]$, and the $m$-vector $g$ is $\left(g_{1} g_{2} \cdots g_{m}\right)^{\mathrm{T}}$.

Relations (19a) and (19b) constitute a new reformulation of Lagrangian dynamics as a constrained quadratic minimization problem. It is interesting to note that the entirety of Lagrangian dynamics is encapsulated in these two relatively simple relations.

Solution of the quadratic programming problem. The solution to the minimization problem posed by Eqs. (19a) and (19b) can be explicitly provided as follows. Using Eq. (19b) we can obtain the vector $\ddot{q}$ as

$$
\ddot{q}=D^{-} g+\left(I-D^{-} D\right) h,
$$

where the $n \times m$ matrix $D^{-}$is any generalized inverse ( $g$-inverse) of $D$ which satisfies the relation

$$
D D^{-} D=D,
$$

and the vector $h$ is arbitrary [8]. Substituting relation (20) in (19a) we thus need to find the vector $h$ such that we obtain

$$
\operatorname{Min}\|H h-z\|_{2}^{2}
$$

where

$$
H=A^{1 / 2}\left(I-D^{-} D\right)
$$

and

$$
z=-A^{1 / 2}\left\{D^{-} g+A^{-1}(f-Q)\right\} .
$$

The solution to the least-squares problem posed in (22) can be expressed as [8]

$$
h=H_{\mathrm{ls}}^{-} z+\left(I-H_{\mathrm{ls}}^{-} H\right) w,
$$

where $w$ is again an arbitrary vector and $H_{\mathrm{ls}}^{-}$is any "generalized least-squares inverse" of the matrix $H$. This least-squares inverse is defined by the relations

$$
\begin{gathered}
H H_{\mathrm{ls}}^{-} H=H, \\
{\left[H H_{\mathrm{ls}}^{-}\right]^{\mathrm{T}}=H H_{\mathrm{ls}}^{-} .}
\end{gathered}
$$

Using (25) in (20) yields the solution

$$
\begin{aligned}
\ddot{q}= & \left\{D^{-}-\left(I-D^{-} D\right) H_{\mathrm{ls}}^{-} A^{1 / 2} D^{-}\right\} g+\left\{\left(I-D^{-} D\right) H_{\mathrm{ls}}^{-} A^{-1 / 2}(Q-f)\right\} \\
& +\left\{\left(I-D^{-} D\right) w-\left(I-D^{-} D\right) H_{\mathrm{ls}}^{-} H w\right\} .
\end{aligned}
$$

The last expression in braces on the right-hand side is zero, because we can always express $H_{\mathrm{ls}}^{-}$as $\left(I-D^{-} D\right)_{\mathrm{ls}}^{-} A^{-1 / 2}$, where $\left(I-D^{-} D\right)\left(I-D^{-} D\right)_{\mathrm{ls}}^{-}\left(I-D^{-} D\right)=$ $\left(I-D^{-} D\right)$. Hence we obtain the general equations of motion of the constrained system as

$$
\ddot{q}=\left\{D^{-}-\left(I-D^{-} D\right) H_{\mathrm{ls}}^{-} A^{1 / 2} D^{-}\right\} g+\left\{\left(I-D^{-} D\right) H_{\mathrm{ls}}^{-} A^{-1 / 2}(Q-f)\right\} .
$$


In general, the elements of the matrices $A, D$, and $H$ are functions of $q$ and $t$; the vectors $f$ and $Q$ are functions of $q, \dot{q}$, and $t$.

We note that the inverses $D^{-}$and $H_{\mathrm{ls}}^{-}$are, in general, two different types of inverses: the first is any $g$-inverse of $D$, the second is any least-squares $g$-inverse of $H$. The expression (29) leaves the actual choice of these inverses open. The Moore-Penrose (MP) inverse [8] is a subset of each of these inverses and may be used for both $D^{-}$and $H_{\mathrm{ls}}^{-}$. It has the advantage of being unique and of being readily available in many computing environments. Both Eqs. (28) and (29) would therefore be valid when the MP inverse replaces the generalized inverses indicated in these equations. In particular, we observe that Eq. (29) then leads to the fundamental equations of motion

$$
\ddot{q}=\left\{D^{+}-\left(I-D^{+} D\right) H^{+} A^{1 / 2} D^{+}\right\} g+\left\{\left(I-D^{+} D\right) H^{+} A^{-1 / 2}(Q-f)\right\},
$$

where the superscript " + " is used to denote the unique MP generalized inverse. Here $D^{+}$is the MP inverse of $D$ and $H^{+}$is the MP inverse of $A^{1 / 2}\left(I-D^{+} D\right)$.

Despite the different choices of the inverses in Eq. (29), since the matrix $A$ is positive definite, the solution of the constrained minimization problem (19) exists and is unique as long as the equation set $(19 \mathrm{~b})$ is consistent [9]. We note that this solution (given by either of Eqs. (29) or (30)) is unique even when the rank of $D$ is less than $m$, as long as the system of equations (19b) is consistent and $A$ is nonsingular.

When there are no constraints on the mechanical system, the solution to the least squares problem represented by $(19 a)$ is naturally given by

$$
\ddot{q}=A^{-1}(Q-f),
$$

as Eq. (19b) no longer exists. Alternately, one can obtain this result from Eq. (29) by using the $m$ independent constraint equations (19b) with the $m \times n$ matrix $D=0$ and the vector $g=0$. In this case $D^{-}=0$ and $H_{\mathrm{ls}}^{-}=A^{-1 / 2}$.

Connections with the $L 1$ and $L 2$ formulations. We now explore the connections which our new system of equations for constrained, discrete mechanical systems has with the L1 and L2 formulations. In particular we are interested in two things. Firstly, can we explicitly determine the forces of constraint on the system generated by virtue of the system being constrained by the equation set (2)?; and, secondly, knowing Eq. (29) can we obtain explicitly the Lagrange multipliers $\lambda_{r}$ in Eq. (5) directly? Both these aspects will provide a deeper understanding of the results obtained so far. In addition, the first aspect is important, from an applications standpoint, in determining the control forces required to be provided for a system to satisfy a given set of constraints. Such situations occur often in practical situations when one wants, say, a robot-arm or a precision tool to follow a prescribed trajectory.

(a) Explicit determination of the control forces. Noting that the effect of the constraints provided by Eq. (2) is to provide a set of constraining forces on the mechanical system, we can express the equations of motion of the constrained system as

$$
\ddot{q}=A^{-1}\left(Q+Q^{\prime}-f\right),
$$


where the generalized force vector contributed by the constraints is represented, as before, by $Q^{\prime}$. Comparing Eqs. (29) and (32) we obtain

$$
Q^{\prime}=\left(I-A X A^{-1}\right)[f(q, \dot{q}, t)-Q]+A(I-X) D^{-} g,
$$

where we have denoted by the matrix $X$ the quantity $\left(I-D^{-} D\right) H_{\mathrm{ls}}^{-} A^{1 / 2}$.

Thus we have explicitly determined the control force $Q^{\prime}$ required for the mechanical system to "follow" the constraints given by Eq. (2). We note that this force, in general, constitutes a feed-back control force since it depends on $q(t), \dot{q}(t)$, and $t$. Furthermore, the control can be split as a linear combination of two components, one of which varies linearly with the vector $g$.

(b) Explicit determination of Lagrange multipliers. We observe from the equation set (5) that the constraint forces are given by the relation

$$
D^{\mathrm{T}} \Lambda=Q^{\prime},
$$

where the $m$-vector $\Lambda$ of Lagrange multipliers is defined by $\Lambda:=\left[\lambda_{1} \lambda_{2} \cdots \lambda_{m}\right]^{\mathrm{T}}$. The vector $Q^{\prime}$ is the constraint force $n$-vector. Noting that matrix $D$ has rank $m$ (since we assume, for the L2 formulation, that the $m$ constraints are independent), the $m \times m$ matrix $\left(D D^{\mathrm{T}}\right)$ is nonsingular because $D^{\mathrm{T}}$ has all its $m$ columns linearly independent. Multiplying both sides of Eq. (34) by $D$, and using Eq. (33), we obtain the Lagrange multipliers explicitly as

$$
\Lambda=\left(D D^{\mathrm{T}}\right)^{-1} D Q^{\prime}=\left(D D^{\mathrm{T}}\right)^{-1} D\left\{\left(I-A X A^{-1}\right)[f(q, \dot{q}, t)-Q]+A(I-X) D^{-} g\right\} .
$$

These multipliers have thus been obtained in closed form for general constraints which need not be known a priori. We note that they are, in general, functions of $q(t), \dot{q}(t)$, and $t$.

Illustrative examples. We consider two examples of nonholonomic systems. We illustrate the applicability of our results by explicitly determining the differential equations of motion of these constrained systems. These examples have been taken from Rosenberg's book [2, pp. 240 and 258, respectively].

1. Consider a particle moving in three-dimensional euclidean space, free of any "given" forces. Let its position at any time $t$ be denoted by $(x, y, z)$. The particle is subjected to the nonholonomic constraint $\dot{y}=z \dot{x}$. The initial conditions are specified so that at time $t=0$, the particle satisfies this constraint. Determine the accelerations of the particle so that it always satisfies the constraint; or, alternatively, find the constraint forces so that the particle satisfies the constraint?

Here, $\left(q_{1}=x, q_{2}=y, q_{3}=z\right)$, and the particle is subjected to the nonholonomic catastatic constraint

$$
\dot{y}=z \dot{x},
$$

and the "given" forces are all zero. The system has two degrees of freedom; yet, the nonholonomic nature of the constraint requires three coordinates for a specification of the system's configuration.

The matrix $D=\left[\begin{array}{lll}-z & 1 & 0\end{array}\right]$ here, and the matrix $D^{-}=\frac{r}{\left(1+z^{2}\right)}\left[\begin{array}{lll}-z & 1 & 0\end{array}\right]^{\mathrm{T}}$. The matrix $A$ and the vector $g$ are scalars equal to unity and $\dot{z} \dot{x}$ respectively. The vectors 
$f$ and $Q$ are zero, and the MP inverse of $\left(I-D^{-} D\right)$, obtained by using full rank factorization, is given by

$$
\left(I-D^{-} D\right)^{+}=\left[\begin{array}{ccc}
\frac{1}{\left(1+z^{2}\right)} & \frac{z}{\left(1+z^{2}\right)} & 0 \\
\frac{z}{\left(1+z^{2}\right)} & \frac{z^{2}}{\left(1+z^{2}\right)} & 0 \\
0 & 0 & 1
\end{array}\right] .
$$

Using Eq. (29) we obtain explicitly the constrained equations of motion as

$$
\left[\begin{array}{c}
\ddot{x} \\
\ddot{y} \\
\ddot{z}
\end{array}\right]=\frac{\dot{z} \dot{x}}{\left(1+z^{2}\right)}\left[\begin{array}{c}
-z \\
1 \\
0
\end{array}\right]
$$

which are of course the same as Rosenberg's result [2, p. 240]. From this expression the constraint forces can be easily obtained.

2. Consider a particle of constant mass $m$, moving in two-dimensional euclidean space. The components of the "given" forces acting on it are $X$ and $Y$. The particle is constrained to move so that $\dot{x}-t \dot{y}=\alpha(t)$. The initial conditions are prescribed so that at time $t=t_{0}$, the particle satisfies the constraint. What are the equations of motion for the system for $t \geq t_{0}$ ? What is the constraint force needed to guide the particle so that it satisfies the constraint?

The system has only one degree of freedom, yet we need two coordinates to specify its configuration because the particle satisfies the acatastatic constraint,

$$
\dot{x}-t \dot{y}=\alpha(t),
$$

where $\alpha$ is a given function of time. The "given" forces are prescribed a priori to be $X$ and $Y$.

Here, the matrix $D=[1-t]$, the vector $g=[\dot{y}+\dot{\alpha}]$, and $D^{-}=\frac{1}{\left(1+t^{2}\right)}[1-t]^{\mathrm{T}}$. The MP inverse of $H$ is given by

$$
H^{+}=\frac{m^{-1 / 2}}{\left(1+t^{2}\right)}\left[\begin{array}{cc}
t^{2} & t \\
t & 1
\end{array}\right]
$$

and the equations of motion of the constrained system are easily obtained by using Eq. (29) as

$$
\left(1+t^{2}\right)\left[\begin{array}{c}
m \ddot{x} \\
m \ddot{y}
\end{array}\right]=\left[\begin{array}{ll}
t^{2} & t \\
t & 1
\end{array}\right]\left[\begin{array}{l}
X \\
Y
\end{array}\right]+m(\dot{y}+\dot{\alpha})\left[\begin{array}{c}
1 \\
-t
\end{array}\right] .
$$

Reference [2], p. 258, has considered the case when $\alpha$ in Eq. (39) is a constant. For this case, the result in [2], after some algebra, is identical to that given by Eq. (41).

The control force required to be applied to "guide" this particle along the trajectory described by Eq. (39), is obtained explicitly, using Eq. (33), as

$$
\left[\begin{array}{l}
Q_{x}^{\prime} \\
Q_{y}^{\prime}
\end{array}\right]=-\frac{1}{\left(1+t^{2}\right)}\left[\begin{array}{cc}
1 & -t \\
-t & t^{2}
\end{array}\right]\left[\begin{array}{l}
X \\
Y
\end{array}\right]+\frac{m(\dot{y}+\dot{\alpha})}{\left(1+t^{2}\right)}\left[\begin{array}{c}
1 \\
-t
\end{array}\right]
$$

Conclusions. In this paper we expose the connections between four seemingly disparate topics-Lagrangian mechanics, Gauss's Principle, quadratic programming, 
and generalized inverses of matrices-and thereby obtain the explicit equations of motion for constrained, discrete mechanical systems. We consider systems encountered within the general framework of Lagrangian mechanics.

Starting with formulation L1 we have, specifically

(1) recast Lagrangian mechanics as a constrained quadratic minimization problem; and

(2) obtained the explicit solution of this quadratic minimization problem through the use of the theory of generalized inverses of matrices.

In addition to the determination of the equations of motion, the development has led to the explicit determination of

(a) the forces of constraint that the system is subjected to, and

(b) the Lagrange multipliers that are germane to formulation L2.

Below we discuss some of the main features of our results, comparing them with previous approaches.

1. One of the main advantages of Lagrangian mechanics is that it provides an explicit formulation for the equations of motion for holonomic systems in terms of an undefined set of generalized coordinates (i.e., no prior selection of a specific set of coordinates is required to formulate the problem). The new approach provided here extends this concept to the explicit formulation of the equations of motion for both holonomic and nonholonomic mechanical systems in terms of also an undefined set of constraint equations (i.e., no prior selection of a specific set of constraint equations is required to yield the explicit equations of motion).

We circumvent the need to use the Lagrange formulation L2 and the consequent need to determine the Lagrange multipliers, thereby creating a general formalism that is not dependent on the specific set of constraint equations defining the problem. This new feature has not been available to date, we think, in the presently available approaches in analytical mechanics; in fact, its absence is responsible for the severe bottle-neck in our ability to handle large-order nonholonomically constrained systems.

2. It is worthwhile comparing the formulation presented here with the famed Gibbs-Appell equations [3, 4]. These equations, which were first published in 1899, are considered by Pars as (see [3, p. 202]) "probably the simplest and most comprehensive form of the equations of motion so far discovered." The differential equation formulation of discrete, constrained systems presented in this paper appears to be superior to the Gibbs-Appell formulation both in terms of its utility and its aesthetic.

In the Gibbs-Appell approach, instead of using a set of Lagrange multipliers to handle the Lagrange formulation $\mathrm{L} 1$, the set of coordinates used to describe the system is expanded. An additional $p$ quasicoordinates are used. These quasi-coordinates are related, through nonintegrable Pfaffian forms, to the coordinates that define the system's configuration. This expanded set of coordinates, along with the constraint equations, are then used to express all the coordinates in terms of a set of any $k$ coordinates where $k$ represents the number of degrees of freedom of the system. The Gibbs-Appell equations of motion are then obtained in terms of these privileged 
$k$ coordinates [3]. As pointed out by Pars, and by Neimark and Fufaev (see [3] and [5]), the choice of the quasi-coordinates, the subsequent elimination procedure, as well as the determination of the generalized forces corresponding to these $k$ privileged coordinates, is problem specific; these cannot be determined without knowledge of the specific constraints present. Thus the Gibbs-Appell formulation, which requires a felicitous choice of quasi-coordinates for each specific problem situation, lacks the generality and directness of the present formulation which is applicable to systems where the constraints need not be specified a priori; all we require is that they be expressible in Pfaffian form.

Our direct approach, which explicitly yields the equations of motion, does not require us to expand the set of coordinates and thus averts the need for choosing the additional quasi-coordinates (which must then be followed by the elimination procedure, etc.). Moreover, it is symmetric in that it does not rely on the creation of a "privileged" set of $k$ coordinates, in whose terms the entire system's dynamics is then expressed.

3. We provide the necessary differential equations of motion in terms of the coordinates originally chosen to specify the configuration of the system. For nonholonomically constrained systems, the number of these coordinates exceeds the number of degrees of freedom. No privileged set of coordinates (equal to the number of degrees of freedom) is used here. Our results therefore may have a greater physical and intuitive appeal, as explicit differential equations for the constrained system are provided, directly describing the time-evolution of each of the coordinates originally used to describe the system's configuration. This may lead to an improved physical understanding of the dynamics of the system, an additional advantage over the Gibbs-Appell formulation.

4. The approach to analytical dynamics provided here handles all the different types of constraints with equal ease. Thus the approach is valid for holonomic, nonholonomic, scleronomic, rheonomic, catastatic, and acatastatic constraints. The somewhat artificial classification of constraints in this fashion, brought about by the previous inability of Lagrangian mechanics to provide the explicit equations of motion in terms of a set of constraints that are not specified from the start, is therefore made unnecessary, and perhaps obsolete. An important aspect of our results is that the procedure provided here can be easily automated so as to handle the dynamics of general systems with general constraints.

5. The approach provides a general formulation for determining the "control, or constraint, forces" required to cause a system to satisfy a set of holonomic and/or nonholonomic constraints. This set of constraints does not need to be specified prior to the formulation. This aspect of our results has important applications in the areas of active control of structural and mechanical systems. We anticipate its imminent use for systems, like tall buildings subjected to strong earthquake ground shaking, and for robot-arms and machine tools which require to be "guided" so that they follow a given trajectory, or more generally, satisfy a given set of constraints.

6. The approach presented here has deep connections to the Lagrange multiplier formulation (referred to as formulation L2). Using our results one can obtain the 
Lagrange multipliers explicitly, again, in terms of an undefined set of constraints.

7. Very often in large-order systems with a large number of constraints (i.e., when $m$ is large), it is difficult to establish the linear independence of the constraint equations. This sometimes becomes especially troublesome when dealing with nonholonomic constraints. The explicit equations of motion obtained here are valid even when these constraint equations are not independent, as long as they are consistent.

8. The formulation of analytical dynamics presented here is somewhat more general and can handle mechanical systems that fall outside the usual preview of Lagrangian dynamics [5]. For instance, for one-sided constraints (e.g., constraints that express impenetrability across a surface, and configuration constraints [2]) which are typically generated by inequality constraints, the quadratic programming problem defined by (P1) is still valid with the expression for $G$ being the same as in Eq. (17). Some of the constraint equations, however, may now be inequalities. The solution of this constrained quadratic problem will yield the motion of the mechanical system. Such extensions of our primary results, we think, will be fruitful areas for future research and will be addressed in future communications.

Note added in proof. Due to the lapse in time between the submission of this paper and its appearance in print the authors point out that the following later paper may be of interest to the reader: "On Motion", by F. E. Udwadia and R. E. Kalaba, Journal of the Franklin Institute, vol. 330, no. 3, pp. 571-577, 1993.

\section{REFERENCES}

[1] J. L. Lagrange, Mécanique Analytique, MMe Ve Courcier, Paris, 1811

[2] R. Rosenberg, Analytical Dynamics of Discrete Systems, Plenum Press, 1972

[3] L. A. Pars, $A$ Treatise on Analytical Dynamics, Ox Bow Press, Connecticut, 1979

[4] P. Appell, Sur une forme générale des équations de la dynamique, Mem. Sci. Math., GauthierVillars, Paris, 1925

[5] Ju. I. Neimark and N. A. Fufaev, Dynamics of Nonholonomic Systems, vol. 33, Amer. Math. Soc., Providence, RI, 1972

[6] C. Gauss, Uber ein neues allgemeines Grundgesetz der Mechanik, J. Reine Angew. Math. 4, 232-235 (1829)

[7] E. Whittaker, $A$ Treatise on the Analytical Dynamics of Particles and Rigid Bodies, Cambridge, 1917

[8] C. Rao and S. Mitra, Generalized Inverse of Matrices and Its Applications, John Wiley, New York, 1972

[9] C. Lawson and R. Hanson, Solving Least Squares Problems, Prentice Hall, Englewoods Cliffs, NJ, 1974 